TRANSACTIONS OF THE

AMERICAN MATHEMATICAL SOCIETY

Volume 352, Number 8, Pages 3623-3638

S 0002-9947(00)02566-6

Article electronically published on March 15, 2000

\title{
GEOMETRIC FLOW AND RIGIDITY ON SYMMETRIC SPACES OF NONCOMPACT TYPE
}

\author{
INKANG KIM
}

\begin{abstract}
In this paper we show that, under a suitable condition, every nonsingular geometric flow on a manifold which is modeled on the Furstenberg boundary of $X$, where $X$ is a symmetric space of non-compact type, induces a torus action, and, in particular, if the manifold is a rational homology sphere, then the flow has a closed orbit.
\end{abstract}

\section{INTRODUCTION}

In this paper we try to give a positive answer to the Seifert conjecture, namely that a smooth flow has a closed orbit or a fixed point, for a manifold which is more rigid in a geometric sense, and for a flow which preserves the geometric structure. As a model space, we choose the Furstenberg boundary of a symmetric space of noncompact type, and as a geometric structure, we choose the whole isometry group of the symmetric space. We will give some information about the geometry of symmetric spaces and the action of isometries on the Furstenberg boundary.

In this direction $\mathrm{H}$. Hofer solved the Weinstein conjecture for $S^{3}$ - every Reeb flow associated to the contact structure on $S^{3}$ has a closed orbit [10]. In the general setting, the Seifert conjecture is disproved in [13] for the $C^{\infty}$ case. The first counterexample for the $C^{1}$ case is given in [16], and later for the $C^{2}$ case in [7].

The $(G, X)$ structure for the manifold $M$ is, briefly speaking, a set of coordinate charts $\left\{\phi_{i}: U_{i} \rightarrow X\right\}$ such that transition functions are in the group $G$. A geometric flow on $M$ is a flow whose one-parameter group preserves this given geometric structure.

The theorem we want to prove is

Theorem 1. A nonsingular geometric flow on a smooth manifold which is a rational homology sphere and is modeled on the Furstenberg boundary of a symmetric space of noncompact type, under suitable assumptions, has a closed orbit.

In [11], the theorem is proved in real, complex and quaternionic hyperbolic spaces. This theorem is a generalization to any symmetric space of noncompact type.

Received by the editors March 12, 1998.

1991 Mathematics Subject Classification. Primary 51M10, 57S25.

Key words and phrases. Symmetric space, geometric flow.

Partially supported by the KOSEF grant 981-0104-021-2.

(C)2000 American Mathematical Society 


\section{2. $(G, X)$ StRUCTURE ON A MANIFOLD}

A pseudogroup $G$ on the space $X$ is a set of homeomorphisms between open sets of $X$. A $(G, X)$-manifold $M$ is a topological space with a $G$-atlas on it. A $G$-atlas is a collection of $G$-compatible coordinate charts whose domains cover $M$. A manifold is a $\left(G, \mathbb{R}^{n}\right)$-space, where $G$ is the set of local homeomorphisms. When $G$ is the set of local diffeomorphisms, it is a differentiable manifold. The most important examples are when $G$ is a Lie group and $X$ is a homogeneous space on which $G$ acts analytically. In our case, $X$ is a symmetric space of non-compact type and $G=I \operatorname{so}(X)$, which is a semi-simple Lie group if $X$ does not have a Euclidean factor. When the rank of the symmetric space is one, there are only four symmetric spaces, namely real, complex, quaternionic and Cayley hyperbolic spaces.

Let $\left\{U_{i}\right\}$ be coordinate charts for $M$, with maps $\phi_{i}: U_{i} \rightarrow X$ and transition functions

$$
\gamma_{i j}=\phi_{i} \circ \phi_{j}^{-1}: \phi_{j}\left(U_{i} \cap U_{j}\right) \rightarrow \phi_{i}\left(U_{i} \cap U_{j}\right) .
$$

If $\alpha$ is a loop based at $x_{0}$, we subdivide $\alpha$ at points $x_{0}=\alpha(0), x_{1}=\alpha\left(t_{1}\right), \cdots$, $x_{n}=\alpha\left(t_{n}=1\right)$, so that each subpath is contained in the domain of a single coordinate chart $\left(U_{i}, \phi_{i}\right)$. As we go along $\alpha$, we successively adjust each chart $\phi_{i}$ so it agrees with the previously adjusted $\phi_{i-1}$ in a neighborhood of $x_{i} \in U_{i} \cap U_{i-1}$. Then the last adjusted chart is

$$
\xi=\gamma_{01} \gamma_{12} \cdots \gamma_{n-1, n} \phi_{n} .
$$

In this way we get a holonomy representation

$$
\rho: \pi_{1}(M) \rightarrow G
$$

Definition 1. For a fixed base point and initial chart $\phi_{0}$, the developing map is the map dev $: \tilde{M} \rightarrow X$ that agrees with the analytic continuation of $\phi_{0}$ along each path, in a neighborhood of the path's endpoint.

Then it is easy to see that

$$
\operatorname{dev} \circ T_{\alpha}=\rho(\alpha) \circ \operatorname{dev},
$$

where $T_{\alpha}$ is the covering transformation corresponding to $\alpha$.

We say that $M$ is a complete $(G, X)$-manifold if dev $: \tilde{M} \rightarrow X$ is a covering map, and $M$ is pseudo-complete if $\operatorname{dev}: \tilde{M} \rightarrow \operatorname{dev}(\tilde{M})$ is a covering map. Note that if $M$ is a closed $(G, X)$-manifold and $G$ is a Lie group acting transitively on $X$ with compact stabilizer $G_{x}$ for some $x$, then $M$ is complete. The reason is that by pulling back the $G$-invariant metric on $X$ by dev, we get a geodesically complete metric on $\tilde{M}$, so a local isometry from a complete Riemannian manifold is always a covering map. See [17]. This is the starting point of our argument in proving the main theorems.

\section{HADAMARD MANIFOLD}

Definition 2. A connected, simply connected complete Riemannian manifold of nonpositive curvature is called an Hadamard manifold.

For subsets $A, B \subset X$ we define the Hausdorff distance $H d(A, B)=\inf \{r>$ $\left.0 \mid A \subset N_{r}(B), B \subset N_{r}(A)\right\}$, where $N_{r}(A)$ denotes the $r$-neighborhood of $A$. Two geodesics $c_{1}, c_{2}$ are parallel if $H d\left(c_{1}(\mathbb{R}), c_{2}(\mathbb{R})\right)$ is finite. 
The following is a very elementary but frequently used technique in Hadamard manifold theory. We record a proof for the reader's sake. It is also proved in [5], for example.

Lemma 1. Let $X$ be a Hadamard manifold. If $c_{1}, c_{2}$ are parallel with $H d\left(c_{1}, c_{2}\right)=$ $a$, then there is an isometric and totally geodesic embedding $\phi: \mathbb{R} \times[0, a] \rightarrow X$ such that $\phi(\mathbb{R} \times 0)=c_{1}(\mathbb{R}), \phi(\mathbb{R} \times a)=c_{2}(\mathbb{R})$.

Proof. Since $H d\left(c_{1}, c_{2}\right)=a, d\left(c_{1}(t), c_{2}(\mathbb{R})\right) \leq a$, it is easy to see that the function $t \rightarrow d\left(c_{1}(t), c_{2}(\mathbb{R})\right)$ is a bounded convex function, so a constant function $a$. By the same argument $d\left(*, c_{1}(\mathbb{R})\right)$ is a constant function $a$ on $c_{2}$. Define $\phi: \mathbb{R} \times[0, a] \rightarrow X$ by $\phi(t, s)=c_{t}(s)$, where $c_{t}:[0, a] \rightarrow X$ is the geodesic from $c_{1}(t)$ to the orthogonal projection of $c_{1}(t)$ on $c_{2}$. Then four geodesics $c_{1}, c_{2}, c_{t_{1}}, c_{t_{2}}$ form a quadrilateral with angles all greater than or equal to $\pi / 2$ by the first variation of arc length. More precisely, if $c(s)$ is a geodesic parameterized by arc length running from a point on $c_{1}$ to its orthogonal projection $p$ on $c_{2}$, let $c_{t}(s)=H(t, s)$ be its variation with $c_{t}(0)$ fixed. Then, by the first variation of arc length,

$$
\frac{d}{d t} L\left(c_{t}\right)=\left\langle T_{(0,1)} H \cdot \frac{\partial}{\partial t}, c^{\prime}(1)\right\rangle \geq 0,
$$

so the angle at $p$ is greater than $\pi / 2$. But since the manifold is non-positively curved, the sum of angles of quadrilateral is less than $\pi / 2$, so all the angles are $\pi / 2$ and the quadrilateral bounds a totally geodesic Euclidean rectangle.

There is another important concept about the Hadamard manifold, namely the ideal boundary. It is the equivalence class of geodesic rays which are asymptotic. Specifically, when the Hamamard manifold is symmetric, its ideal boundary has a Tits building structure. Let $G=I s o^{0}(X)=K A N$ be the Iwasawa decomposition. Geometrically, if $x_{0}$ is a point in $X$, then $K$ is the isotropy group of $x_{0}$, and $A x_{0}$ is the maximal flat passing through $x_{0}$. If $\Lambda^{+}$is the set of positive roots, then the $A^{+} x_{0} \subset A x_{0}$ corresponding to $\Lambda^{+}$is called a Weyl chamber. If

$$
\mathcal{G}=\mathcal{G}_{0} \oplus \sum_{\lambda \in \Lambda} \mathcal{G}_{\lambda}
$$

is the root space decomposition corresponding to a maximal abelian subalgebra $\mathfrak{a}$ where the exponential of $\mathfrak{a}$ is equal to $A$, then $\exp \mathcal{N}^{+}$where $\mathcal{N}^{+}=\sum_{\lambda \in \Lambda^{+}} \mathcal{G}_{\lambda}$, is the nilpotent group $N$. Then the boundaries of all pointed flats form the Tits building tessellating the ideal boundary by Weyl chambers and lower dimensional cells called walls of Weyl chambers.

Note that $A N$ fixes $\overline{A^{+} x_{0}(\infty)}$ pointwise and $M \subset K$ fixes $A^{+} x_{0}(\infty)$ pointwise. In general, if $\Theta$ is the subset of the fundamental system $\Gamma$ of positive roots, it determines a unique face of $\mathcal{A}^{+}$, which is

$$
f_{\Theta}=\{H \in \mathcal{A} \mid \alpha(H)>0, \forall \alpha \in \Gamma-\Theta, \alpha(H)=0, \forall \alpha \in \Theta\} .
$$

If we set $M_{\Theta} \subset K$ to be a stabilizer of $f_{\Theta}$, then $P_{\Theta}=M_{\Theta} A N$ is the stabilizer of $f_{\Theta}$. Then $G / P_{\Theta}$ is the set of all equivalence classes of the face $f_{\Theta}$. When $\Theta=\emptyset$, $P=P_{\Theta}=M A N$ and $G / P$ is the set of Weyl chambers on the ideal boundary. This is called the Furstenberg boundary and we will use this boundary as the model space for the higher rank case. Since $G / P$ is identified with $K / M$, there is a $K$-invariant Riemannian metric on the (Furstenberg) boundary. We will call this complete metric spherical by abuse of terminology. This fact will be used in proving later theorems. 
One last thing one should be familiar with is the horosphere. Given a geodesic $c(t)$, the function

$$
h_{c}(x)=\lim _{t \rightarrow \infty}(d(x, c(t))-t)
$$

is called the Busemann function. Then its level sets are called the horospheres based at $c(\infty)$. This Busemann function is a convex $C^{1}$ function with $\left\|\operatorname{grad} h_{c}\right\|=1$. For more information, see 3 .

\section{RANK ONE SYMMETRIC SPACES}

4.1. The boundary of the rank one symmetric space. The boundary of a rank one symmetric space $X$ is a one point compactification of the nilpotent group $N$ in the Iwasawa decomposition of $G=I s 0^{0}(X)=K A N$, denoted by $N \cup \infty$. An element of $N$ is denoted by $[t, k]$, where $t \in \operatorname{Im} \mathbb{F}$ and $k \in \mathbb{F}, \mathbb{F}$ being real $\mathbb{R}$, complex $\mathbb{C}$, quaternionic $\mathbb{H}$ or Cayley number $\mathbb{O}$. The multiplication is defined by

$$
[t, k]\left[t^{\prime}, k^{\prime}\right]=\left[t+t^{\prime}+2 \operatorname{Im}\left\langle k, k^{\prime}\right\rangle, k+k^{\prime}\right] .
$$

See [12, 14] for references.

There is a sub-Riemannian metric defined on $\partial H_{\mathbb{F}}^{n}$ which inherits from the metrics on the horospheres based at $\infty$. This sub-Riemannian metric, which is known as the Carnot-Carathéodory metric, is $\lim _{t \rightarrow \infty} e^{-2 t} g_{t}$, where $g_{t}$ is a Riemannian metric on the horosphere $H_{t}$ when we foliate $H_{\mathbb{F}}^{2}$ as a one-parameter family of horospheres $H_{t}$ based at $\infty$. Each horosphere has horizontal and vertical distributions. The growth of a vector along a Jacobi field in the horizontal in the distribution is by the factor of $e^{t}$, and the growth of a vector along a Jacobi field in vertical distribution is by the factor of $e^{2 t}$. So when we take the limit of $e^{-2 t} g_{t}$, the length in the vertical direction goes to infinity. Note here that $N$ acts as an isometry group with respect to the Carnot metric, since each orbit of a point by $N$ is a horosphere and $N$ acts isometrically on this horosphere. See [15] for details. We define $\{(0, k) \mid(0, k) \in N\}$, the horizontal set, and $\{(t, 0) \mid(t, 0) \in N\}$, the vertical set.

4.2. Action of isometries on $\partial H_{\mathbb{F}}^{m}$. The action of $I s o\left(H_{\mathbb{F}}^{m}\right)$ extends continuously to $\partial\left(H_{\mathbb{F}}^{m}\right)$. Let $\operatorname{Sim}(N)$ denote the subgroup of $I s o\left(H_{\mathbb{F}}^{m}\right)$ which fixes $\infty$. Then it is isomorphic to $N \rtimes\left(O_{\mathbb{F}}(m-1) \times \mathbb{R}\right)$ for the complex and real hyperbolic cases, $N \rtimes\left(O_{\mathbb{F}}(1) \cdot O_{\mathbb{F}}(m-1) \times \mathbb{R}\right)$ for the quaternionic case, and $N \rtimes(\operatorname{Spin}(7) \times \mathbb{R})$ when the space is Cayley hyperbolic space. For an explicit calculation, set a Cayley number to be a pair of quaternions and define the multiplication by

$$
\left(q_{1}, q_{2}\right)\left(p_{1}, p_{2}\right)=\left(q_{1} p_{1}-\overline{p_{2}} q_{2}, p_{2} q_{1}+q_{2} \overline{p_{1}}\right) .
$$

Also we define $\overline{\left(q_{1}, q_{2}\right)}=\left(\overline{q_{1}},-q_{2}\right)$. Then it satisfies the usual properties such as $x \bar{x}=|x|^{2},|x y|=|x||y|, x^{-1}=\bar{x} /|x|^{2}, \overline{x y}=\bar{y} \bar{x}$. Even though Cayley numbers are not commutative, nor associative, by Artin's lemma a subalgebra generated by two elements is associative. For the next proposition, observe the following calculation: If $\nu$ is a unit imaginary Cayley number, then $[\nu w][z \bar{\nu}]=\nu(w z) \nu^{-1}$. The reason is that $\operatorname{Aut}(\mathbb{O})=G_{2}$ and $G_{2}$ acts transitively on unit imaginaries. The isotropy group of $i$ is a copy of $S U(3)$, and it acts transitively on unit imaginaries orthogonal to $i$. The stabilizer of $i, j$ which fixes $k$ acts transitively on the unit imaginaries orthogonal to $i, j, k$, and it is a copy of $S U(2)$. See []. So using $\operatorname{Aut}(\mathbb{O})$ we can 
assume that $w=(a, 0), z=(b, 0), \nu=(c, r)$, where $a, b, c$ are quaternions and $r$ is a real number. Then a direct calculation shows that

$$
\begin{gathered}
\nu(w z) \nu=\left(c a b c-r^{2} \bar{b} \bar{a}, r c a b+r \bar{b} \bar{a} \bar{c}\right), \\
(\nu w)(z \nu)=\left(c a b c-r^{2} \bar{b} \bar{a}, r b c a+r \bar{a} \bar{c} \bar{b}\right) .
\end{gathered}
$$

But $b c a+\bar{a} \bar{c} \bar{b}=2 \operatorname{Re}(b c a)=2 \operatorname{Re}(c a b)=c a b+\bar{b} \bar{a} \bar{c}$, so $\nu(w z) \nu=(\nu w)(z \nu)$. Since $\nu$ is a unit imaginary, $\bar{\nu}=\nu^{-1}=-\nu$. Now the claim follows from these facts.

Proposition 1. The action of an isometry on the ideal boundary of rank one symmetric space, which fixes 0 and $\infty$, is of the form $[t, z] \rightarrow[\alpha(t), \beta(z)]$, where $\alpha(t)=l^{2} \nu t \nu^{-1}, \beta(z)=l \nu z M$ and $l>0,(\nu, M) \in O_{\mathbb{F}}(1) \times O_{\mathbb{F}}(m-1)$ for $\mathbb{F}=\mathbb{R}, \mathbb{C}, \mathbb{H}$. For the Cayley hyperbolic case, the action belongs to $A \times \operatorname{Spin}(7)$, and the action $[t, z] \rightarrow\left[l^{2} \nu t \nu^{-1}, l \nu z\right]$, where $\nu$ is a unit imaginary Cayley number, is one of those.

Proof. In the Iwasawa decomposition of $I s o(X)=K A N$, the hyperbolic isometries belong to $A \times K$, where $A$ is a maximal abelian subgroup and $K$ is a maximal compact group. Since the space is rank one, $A$ is equal to $\mathbb{R}$. The hyperbolic action is a combination of $[t, z] \rightarrow\left[r^{2} t, r z\right]$ and $[t, z] \rightarrow\left[\nu t \nu^{-1}, \nu z M\right]$. For the Cayley hyperbolic case, we will show that the action $[t, z] \rightarrow\left[\nu t \nu^{-1}, \nu z\right]$ is an isometry. Then, since every bi-Lipschitz map on the ideal boundary comes from an isometry of the space [15], this will finish the proof. The images of two points $[t, z],[s, w]$ under the action are $\left[\nu t \nu^{-1}, \nu z\right]$ and $\left[\nu s \nu^{-1}, \nu w\right]$. Then

$$
\begin{gathered}
d\left(\left[\nu t \nu^{-1}, \nu z\right],\left[\nu s \nu^{-1}, \nu w\right]\right)^{4} \\
=\left|\left[\nu(t-s) \nu^{-1}-2 \operatorname{Im}\langle\nu(w), \nu(z)\rangle,-\nu w+\nu z\right]\right| \\
=\left|\nu(t-s-2 \operatorname{Im}\langle w, z\rangle) \nu^{-1}\right|^{2}+|z-w|^{4} \\
=|t-s-2 \operatorname{Im}\langle w, z\rangle|^{2}+|z-w|^{4} \\
=d([t, z],[s, w])^{4} .
\end{gathered}
$$

\section{5. $\left(I s o\left(H_{\mathbb{F}}^{n}\right), \partial H_{\mathbb{F}}^{n}\right)$ MANIFOLDS AND GEOMETRIC FLOWS}

An $\left(I s o\left(H_{\mathbb{F}}^{n}\right), \partial H_{\mathbb{F}}^{n}\right)$ structure on a manifold $M$ is a geometric structure locally modeled on $\partial H_{\mathbb{F}}^{n}$ with coordinate change lying in $I s o\left(H_{\mathbb{F}}^{n}\right)$. Set $I s o\left(H_{\mathbb{F}}^{n}\right)=G$ and $\partial H_{\mathbb{F}}^{n}=X$. $\operatorname{Aut}(M)$ denotes the group of all $(G, X)$-automorphisms of $M$. Then there is a developing pair

$$
(\rho, \operatorname{dev}):(\operatorname{Aut}(\tilde{M}), \tilde{M}) \rightarrow(G, X) .
$$

$\rho\left(\pi_{1}(M)\right)$ is called the holonomy group of $M$. Since $\operatorname{Aut}(M) \subset \operatorname{Diff}(M)$, there is a map $\phi: \operatorname{Aut}(M) \rightarrow \operatorname{Out}\left(\pi_{1}(M)\right)$. Let $\mathcal{C}_{\text {Aut }(\tilde{M})}\left(\pi_{1}(M)\right)$ be the centralizer of $\pi_{1}(M)$ in $\operatorname{Aut}(\tilde{M})$. Then we have

Lemma 2. The lift of $\operatorname{Aut}(M)^{0}$ to $\tilde{M}$ belongs to $\mathcal{C}_{A u t(\tilde{M})}\left(\pi_{1}(M)\right)$. 
Proof. Let $\alpha \in \operatorname{Aut}(M)^{0}$, and let $\tilde{\alpha}$ be its lift to $\tilde{M}$. Then, for any $\beta \in \pi_{1}(M)$,

$$
\tilde{\alpha} \circ \beta=\alpha_{*}(\beta) \circ \tilde{\alpha}=\beta \circ \tilde{\alpha}
$$

since $\alpha_{*}=1$ on $\pi_{1}(M)$. To see the above equality more precisely, let $x_{0} \in M$, and $\tilde{x_{0}}$ is the point in $\tilde{M}$ which covers $x_{0}$. Then $\tilde{\alpha}\left(\tilde{x_{0}}\right)$ be the point covering $\alpha\left(x_{0}\right)$. Since $\alpha$ is homotopic to the identity, if we denote the loop corresponding to $\beta$ by $l_{\beta}(t)$, then $l_{\beta}(t)$ is homotopic to $\alpha\left(l_{\beta}(t)\right)$ by $H(t, s):[0,1] \times[0,1] \rightarrow M$ such that $H(t, 0)=l_{\beta}(t)$ and $H(t, 1)=\alpha\left(l_{\beta}(t)\right)$. Note that $\beta\left(\tilde{x_{0}}\right)=\tilde{l}_{\beta}(1)$, where $\tilde{l}_{\beta}$ is a lift of $l_{\beta}(t)$ starting from $\tilde{x_{0}}$. If $\tilde{H}(t, s)$ denotes the lift of $H(t, s)$ such that $\tilde{H}(t, 1)=\tilde{\alpha}\left(\tilde{l}_{\beta}\right)(t)$ which is a lift of $\alpha\left(l_{\beta}(t)\right)$, then $\tilde{H}(t, 0)$ is a lift of $l_{\beta}(t)$. Note that $\beta(\tilde{H}(0,0))=\tilde{H}(1,0)$. Since $\beta$ is a homeomorphism, $\beta(\tilde{H}(0, s))=\tilde{H}(1, s)$ for all $s$ near zero. Also, given $0 \leq s \leq 1$, there is a unique $\gamma_{s} \in \pi_{1}(M)$ such that $\gamma_{s}(\tilde{H}(0, s))=\tilde{H}(1, s)$. Since this map $s \rightarrow \gamma_{s}$ is locally constant, $\gamma_{1}=\beta$, i.e $\beta\left(\tilde{\alpha}\left(\tilde{x_{0}}\right)\right)=\tilde{\alpha}\left(\beta\left(\tilde{x_{0}}\right)\right)$. This shows that $\alpha_{*}=$ the identity on $\pi_{1}(M)$.

A flow whose one-parameter group $\left\{\phi_{t}\right\}_{t \in \mathbb{R}}$ is in $\operatorname{Aut}(M)$ is called a geometric flow on $M$. One should also note that the lift of $\operatorname{Aut}(M)$ in $\operatorname{Aut}(\tilde{M})$ is the normalizer of $\pi_{1}(M)$ in $\operatorname{Aut}(\tilde{M})$.

The following proposition is crucial to proving the main theorem.

Proposition 2. Let $\left\{\phi_{t}\right\}$ be a closed noncompact one-parameter group of

$$
\mathcal{C}_{\text {Iso }\left(H_{\mathbb{F}}^{n}\right)}\left(\rho\left(\pi_{1}(M)\right)\right) \text {. }
$$

Then one of the following is true:

(1) $M$ is $(G, X)$-equivalent to $X$.

(2) $\phi_{t}$ fixes a unique fixed point $\{\infty\}$ with $\operatorname{dev}^{-1}(\infty)=\emptyset$.

(3) $\phi_{t}$ fixes exactly two points $\{[0,0], \infty\}$ with $\operatorname{dev}^{-1}(\{[0,0], \infty\})=\emptyset$.

Furthermore, in cases (2) and (3), Aut(M) is compact.

Proof. Let $\rho\left(\pi_{1}(M)\right)=\Gamma$. Suppose $M$ is not $(G, X)$-equivalent to $X$.

Case I. $\phi_{t}$ is hyperbolic.

We show that $\Gamma \subset M A$ in the Iwasawa decomposition of $G=K A N$. Let 0 and $\infty$ be two fixed points of $\phi_{t}$. If $\gamma \in \Gamma$, at least $\gamma^{2}$ will fix 0 and $\infty$, since $\gamma$ commutes with $\phi_{t}$, which is true for all Hadamard manifolds. So we conclude, up to an index 2 subgroup, that $\Gamma \subset M A$, where $M$ is a subgroup of $K$ which fixes 0 and $\infty$.

Then we show that $\operatorname{dev}(\tilde{M}) \cap\{0, \infty\}=\emptyset$. Suppose not. Give a complete metric on $X-\{0, \infty\}$ invariant under $M A$. Then pull back the metric by dev to $\tilde{M}-\operatorname{dev}^{-1}\{0, \infty\}$. Then

$$
\operatorname{dev}^{\prime}: \tilde{M}-\operatorname{dev} v^{-1}\{0, \infty\} \rightarrow X-\{0, \infty\}
$$

will be a covering map. The reason is as follows. Since $\Gamma \subset M A, \pi_{1}(M)$ acts as isometries on $\tilde{M}$. Since $d e v^{-1}\{0, \infty\}$ is left invariant under $\pi_{1}(M)$, it follows that $M-\pi\left(\operatorname{dev}^{-1}\{0, \infty\}\right)$ will inherit the metric. We claim it is complete. It suffices to show that the induced metric on $\tilde{M}-\operatorname{dev}^{-1}\{0, \infty\}$ is complete. Let $\left\{x_{i}\right\}$ be a Cauchy sequence in $\tilde{M}-d e v^{-1}\{0, \infty\}$. Let $x^{\prime}$ be an accumulation point (it exists since $M$ is compact) of $\pi\left(x_{i}\right)$ in $M$, where $\pi: \tilde{M} \rightarrow M$ is a covering map. By choosing a topological neighborhood $N$ of $x^{\prime}$ such that $\bar{N}$ is evenly covered by $\pi$ and $\bar{N}$ is compact, we can see that there is a point $x$ in $\tilde{M}$ so that $\lim x_{i}=x$ (after passing to a subsequence). Since $d e v^{\prime}$ decreases the distance, $\left\{d e v^{\prime}\left(x_{i}\right)\right\}$ is a Cauchy 
sequence. Since the metric on $X-\{0, \infty\}$ is complete, it converges to some point $y$ in $X-\{0, \infty\}$. But since $\lim x_{i}=x$, it follows that $\lim \operatorname{dev}^{\prime}\left(x_{i}\right)=\operatorname{dev}(x)$, and by the uniqueness of the limit point of the Cauchy sequence, $\operatorname{dev}(x)=y$. Let $V$ be a metric ball around $y$ so that $V$ has an isometric neighborhood $\tilde{V}$ in $\tilde{M}-\operatorname{dev} v^{-1}\{0, \infty\}$ which contains $x$. This shows that a Cauchy sequence $x_{i}$ converges to the point $x$ in $\tilde{V}$ with $d e v^{\prime}(x)=y$. This shows that every Cauchy sequence in $\tilde{M}-d e v^{-1}\{0, \infty\}$ converges in the space itself, which implies that the metric is complete. So the metric on $\tilde{M}-d e v^{-1}\{0, \infty\}$ is complete and $d e v^{\prime}$ becomes a Riemannian covering map onto $X-\{0, \infty\}$. When the dimension of $X$ is greater than or equal to 3 , $X-\{0, \infty\}$ is simply connected. So $d e v^{\prime}$ is a homeomorphism. Then it is easy to see that dev is a homeomorphism. When the dimension of $X$ is 2 , by analyzing the universal cover of the surface and using the fact that dev is a local homeomorphism, it can be shown that $d e v$ is a homeomorphism. In any case, if $\operatorname{dev}(\tilde{M}) \cap\{0, \infty\} \neq \emptyset$, then $\pi_{1}(M)$ will have a fixed point since $\Gamma$ has a fixed point, which is not possible for covering transformations.

Since for any $\alpha \in \rho(\operatorname{Aut}(M))$ we have $\alpha \circ \gamma(l)=\gamma^{\prime} \circ \alpha(l)$, where $l$ is a geodesic with end points 0 and $\infty$, we know that any element in the normalizer $N_{G}(\Gamma)$ of $\Gamma$ in $G$ already fixes two points. So any stabilizer of $x$ in $N_{G}(\Gamma)$ where $x$ is in $\partial X-\{0, \infty\}$ is compact. Note that $N_{G}(\Gamma) \subset M A$. Take a Riemannian metric invariant under $M A$. Then, by pulling back the metric to $\tilde{M}$, we know that $A u t(M)=I \operatorname{so}(M)$. Since $M$ is compact, $\operatorname{Aut}(M)$ is compact.

Case II. $\phi_{t}$ is parabolic.

Let $\infty$ be a unique fixed point of $\phi_{t}$. If $\gamma \in \Gamma$, then $\gamma \circ \phi_{t}(\infty)=\phi_{t} \circ \gamma(\infty)$. So $\gamma(\infty)=\infty$ since $\phi_{t}$ has a unique fixed point, which implies that $\Gamma \subset M A N$. Suppose 0 is another fixed point of $\gamma$ different from $\infty$. Then $\gamma \circ \phi_{t}(0)=\phi_{t} \circ \gamma(0)$. So $\phi_{t}(0)$ is a fixed point of $\gamma$. But $\phi_{t}(0) \notin\{0, \infty\}$ for all $t>0$. So $\gamma$ is not a hyperbolic isometry fixing $\infty$, since a hyperbolic isometry fixes only two points. Then $\gamma$ is either a parabolic isometry or an elliptic element fixing $\infty$, so it leaves invariant any horosphere based at $\infty$. If $\gamma=$ man with $a \neq$ identity, $\gamma$ will not leave invariant any horosphere based at $\infty$. This shows that $\Gamma \subset M N$, and $\Gamma$ cannot be in $M$.

Take a Riemannian metric on $X-\infty$ invariant under $M N$. Since $M N$ acts transitively on $X-\infty$, the invariant Riemannian metric is geodesically complete. Pull back the metric to $\tilde{M}$ by dev. Then it is easy to see that this induced metric on $\tilde{M}$ is geodesically complete, and dev becomes a local isometry. Since $\partial X-\infty$ is simply connected, dev is an isometry. So $M$ is homeomorphic to $(X-\infty) / \Gamma$.

Now we want to show that the normalizer (= lift of $\operatorname{Aut}((X-\infty) / \Gamma)$ ) of $\Gamma$ in $G$ has no hyperbolic isometry. Suppose $\alpha$ is a hyperbolic isometry fixing $\infty$ and $P$. If we form a fundamental domain $F$ of $\Gamma$ containing $P, \alpha(F)$ is uniformly expanded or contracted according to whether $P$ is an expanding fixed point or a contracting fixed point of $\alpha$. So $\alpha(F)$ cannot cover $(X-\infty) / \Gamma$ in one-to-one fashion, so $\alpha$ cannot be projected down as an automorphism of $(X-\infty) / \Gamma$. This shows that the normalizer of $\Gamma$ is in $M N$. So

$$
\operatorname{Aut}(M)=\operatorname{Aut}((X-\infty) / \Gamma)=\operatorname{Iso}((X-\infty) / \Gamma),
$$

which is compact.

In this section we prove a theorem in a broader setting, namely in a negatively curved homogeneous manifold. 
According to [1] (see the proof of Proposition 2.5), if $G$ is the identity component of the whole isometry group of $X$, it can be decomposed into $K S$, where $K$ is a maximal compact subgroup (which is the isotropy subgroup of $x_{0}$ ) and $S$ is a simply connected solvable subgroup of $G$ acting simply transitively on $X$. Set $S=A N$ as in $[8$, where $\mathfrak{n}=[\mathfrak{s}, \mathfrak{s}]$. When the algebraic $\operatorname{rank}, \operatorname{dim} \mathcal{A}$, of $X$ is 1 , which is the case since $X$ is negatively curved, Druetta [4] proved that $X$ satisfies the visibility axiom, i.e. any two points in $\partial X$ can be joined by a geodesic. Let $\infty=A x_{0}(\infty)$. Let $\gamma$ be a unique geodesic connecting $\infty$ and $x \in \partial X$. Choose any element $x^{\prime} \in \gamma$. Since $N A$ acts transitively on $X$, there is $n a$ so that $n a\left(x_{0}\right)=x^{\prime}$. Furthermore, $n a\left(A x_{0}\right)$ is a geodesic connecting $\infty$ and $x^{\prime}$, since $N A$ fixes $A x_{0}(\infty)$. This shows that $n\left(A x_{0}(-\infty)\right)=x$. So $N$ acts transitively on $\partial X-\infty$. Note that since $N A$ acts simply transitively on $X, A x_{0}$ becomes a real geodesic connecting $x_{0}$ and $\infty$, and $N x_{0}$ becomes a horosphere based at $\infty$ through $x_{0}$. Let $\gamma_{x}$ be a unique geodesic emanating from $\infty$ and landing at a given point $x \in \partial X . \gamma_{x}$ intersects $N x_{0}$ at a unique point. Since $N$ acts on $N x_{0}$ simply transitively, for any point $y \in \partial X-\infty$ there is a unique $n \in N$ such that $y=\left(n \gamma_{x}\right)(\infty)$, which shows that $N$ acts on $\partial X-\infty$ simply transitively. Then just give a left invariant metric on $\partial X-\infty$ by $M N$, where $M \subset K$ fixes $\infty$.

Next we want find a metric on $\partial X-A x_{0}( \pm \infty)$ invariant under $M A$. Let $S$ be the set of geodesics through $x_{0}$ which are orthogonal to $A x_{0}$. Since $M$ fixes $x_{0}$, it leaves $S$ invariant. Then $\partial S$ is topologically $S^{n-2}$, where $n$ is the dimension of $X$. Now take an $M$-invariant metric on $\partial S$. This is possible since $M$ is compact. Namely take any complete metric and average it by $M$. Transport this metric by $A$ on $\partial X-A x_{0}( \pm \infty)$.

We state the theorem with some hypothesis.

Theorem 2. Let $X$ be a homogeneous Hadamard manifold with negative curvature such that, for any totally geodesic submanifold $Y$ in $X, \operatorname{Stab}(Y)=\{\alpha \in$ Aut $(X) \mid \alpha(Y)=Y\} \subset \operatorname{Aut}(X)$ acts transitively on $Y$. Let $M$ be a compact manifold modeled on $(I s o(X), \partial X)$. Then every nonsingular geometric flow in $\operatorname{Aut}(M)^{0}$ induces a $k$-torus action on $M$. If $M$ is a rational homology sphere, then the flow has a closed orbit.

Proof. We claim that the nonsingular flow $\left\{\xi_{t}\right\}$ must be compact. Suppose it is noncompact. Then its holonomy image $\left\{\phi_{t}\right\}$ must be noncompact for the following reason. Proposition 2 holds in this case also since there are complete invariant metrics on $\partial X-A x_{0}( \pm \infty)$ and $\partial X-\infty$.

Let $G=G_{1} \cdot G_{2}$ be a Levi decomposition, where $G_{1}$ is a radical. If $G_{1}$ is trivial, $G$ becomes a semi-simple Lie group. We divide the proof into two cases.

Case I. $G_{1}$ is not trivial.

In this case there is a global fixed point $\infty$ on $\partial X$. See [8]. So if the holonomy image of the noncompact flow is compact, it is elliptic and it fixes some totally geodesic subspace $Y \subset X$. First we show that there is a metric on $\partial X-\partial Y$ invariant under $A u t(\partial X-\partial Y)$. Let $x_{0} \in Y$, and let $S$ be the set of geodesics through $x_{0}$ which are orthogonal to $Y$. Then $\operatorname{Stab}_{\operatorname{Stab}(Y)}\left(x_{0}\right)=\left\{\alpha \in \operatorname{Stab}(Y) \mid \alpha\left(x_{0}\right)=x_{0}\right\}$ is compact and leaves $S$ invariant. So take the invariant metric under $\operatorname{Stab}_{\operatorname{Stab}(Y)}\left(x_{0}\right)$ on $\partial S$. Then transport this metric on $\partial X-\partial Y$ using the fact that $\operatorname{Stab}(Y)$ acts transitively on $Y$. This shows the existence of the metric on $\partial X-\partial Y$ invariant under $\operatorname{Aut}(\partial X-\partial Y)$. If $\operatorname{dev}(x) \in \partial Y$ for some $x \in \tilde{M}$, then, since $\operatorname{dev} \circ \tilde{\xi}_{t}(x)=\operatorname{dev}(x)$ 
and dev is a local homeomorphism, $\tilde{\xi}_{t}(x)=x$. Then $\xi_{t}$ is singular, so we have a contradiction. So $\operatorname{dev}(\tilde{M}) \cap Y=\emptyset$.

Then the developing pair reduces to

$$
(\rho, \operatorname{dev}):(\operatorname{Aut}(\tilde{M}), \tilde{M}) \rightarrow(\operatorname{Aut}(\partial X-\partial Y), \partial X-\partial Y),
$$

and $\operatorname{Aut}(M)=I s o(M)$ is compact. This is a contradiction.

Case II. $G$ is semi-simple.

Note that, in this case, the homogeneous manifold is a symmetric space of noncompact type of rank one, and $G$ satisfies all the hypothesis in the theorem. See [9], Theorem 7.2. Suppose $x_{0}$ is a unique fixed point of $\phi_{t}$. Take a spherical metric on $\partial H_{\mathbb{F}}^{n}=\partial X$ which is invariant under $K$. Since $\Gamma=\rho\left(\pi_{1}(M)\right)$ commutes with $\phi_{t}, \Gamma \subset K$. Also, $\tilde{\xi}_{t} \subset I s o(\tilde{M})$, since $\phi_{t} \subset K$. Now $M$ inherits the metric from $\tilde{M}$. Since $M$ is closed, $\tilde{M}$ has a complete metric, which makes $\tilde{M}$ and $\partial X$ isometric. Since $I s o(\tilde{M})=I s o(\partial X)$ is compact and $\tilde{\xi}_{t}$ is noncompact, and also $\xi_{t} \subset I s o(M)$ is compact, we have a contradiction.

If $\phi_{t}$ fixes some totally geodesic subspace, the argument is the same as above.

Now we are ready to prove the theorem. When the flow is noncompact, by proposition $2, M$ is $\left(I s o\left(H_{\mathbb{F}}^{n}\right), \partial H_{\mathbb{F}}^{n}\right)$-equivalent to $\partial H_{\mathbb{F}}^{n}$ and the flow is either parabolic or loxodromic. So the flow is singular. This implies that the flow is compact. Since $\operatorname{Aut}(M)^{0}$ is a Lie group, the closure of the one-parameter group induced by the flow is a k-torus $T^{k}$ in $A u t(M)^{0}$. And since the flow is non-singular, $T^{k}$ has no fixed point on $M$. Then by the result of Borel [2] the fixed point set $\Sigma$ of some subtorus $T^{\prime}$ of dimension $k-1$ is nonempty. So the orbit of $T^{k}$ through a point of $\Sigma$ is a circle.

For the detailed exposition of homogeneous manifolds, see [1] and [8].

\section{HighER RANK CASE}

In the higher rank case, the situation is much more complicated.

6.1. Classification of isometries and their properties. For $\gamma \in I \operatorname{so}(X)$, define $d_{\gamma}(x)=d(x, \gamma(x))$. Then there are four kinds of isometries:

(1) Elliptic, if $\gamma$ has a fixed point in $X$.

(2) Hyperbolic, if $d_{\gamma}$ assumes the infimum and $\inf _{x \in X} d_{\gamma}(x)>0$. In this case there is a geodesic, called the invariant geodesic, such that $\gamma$ translates along this geodesic and its translation length is the infimum of $d_{\gamma}$. The set of geodesics parallel to the invariant geodesic is left invariant by $\gamma$.

(3) Pure parabolic, if $d_{\gamma}$ does not assume the infimum, and the infimum is zero.

(4) Mixed parabolic, if $d_{\gamma}$ does not assume the infimum and the infimum is positive.

We call both pure and mixed parabolic elements parabolic. An example of a mixed parabolic element is $(g, h) \in I s o\left(H_{\mathbb{R}}^{2}\right) \times I s o\left(H_{\mathbb{R}}^{2}\right)$, where $g$ is hyperbolic and $h$ is parabolic.

In the symmetric space of higher rank, the isometry group $G$ has the Iwasawa decomposition $K A N$. The following observation is sometimes useful.

Lemma 3. (1) Every hyperbolic isometry $\gamma$ can be conjugated into $K A$.

(2) If $\gamma=k a n$ is parabolic, then each factor $k, a, n$ should leave invariant the horosphere $H$ which $\gamma$ leaves invariant. If a is not in $A x_{0} \cap H$, then $\gamma=k n$, since 
a leaves invariant $A x_{0} \cap H$. Note that $A x_{0} \cap H$ is codimension one in $A x_{0}$, so the latter case is generic. In particular, if the parabolic element is not mixed, it is in $M N$, where $M \subset K$ fixes $A x_{0}(\infty)$.

(3) No parabolic isometry fixes two opposite Weyl chambers.

Proof. (1) Let $l$ be the invariant geodesic of $\gamma$. Fix $x_{0}$ on $l$ and a flat $F$ containing $l$. If $W_{\infty}$ is a Weyl chamber to which $l(\infty)$ belongs, there is a unique Iwasawa decomposition $K A N$ where $K$ is an isotropy group of $x_{0}$ and $F=A x_{0}$ and $N$ is determined uniquely by $x_{0}$ and $W_{\infty}$. Suppose $\gamma=k a n$. Then $k a n(l)=l$. If $n \neq$ the identity, then $n\left(A x_{0}\right) \cap A x_{0}=\emptyset$, and so $a n\left(A x_{0}\right) \cap A x_{0}=\emptyset$ since $a$ leaves $A x_{0}$ invariant and is a homeomorphism on $X$. Since $k\left(x_{0}\right)=x_{0}, k a n(l) \neq l$, which is a contradiction. So $n=$ the identity.

(2) A parabolic isometry $\gamma$ fixes a point $z$ at infinity and leaves invariant any horosphere based at that point, see [3]. Fix a point $x_{0}$ on the horosphere $H$ and a geodesic $l$ connecting $x_{0}$ and $z$. Take an Iwasawa decomposition $K A N$ so that $K=\operatorname{Stab}\left(x_{0}\right)$ and $A x_{0}$ contains $l$ and $z \in \overline{A^{+} x_{0}(\infty)}$. If $\gamma=k a n$, then $n(H)=H$, since $N$ fixes $\overline{A^{+} x_{0}(\infty)}$ pointwise and the orbit $N\left(x_{0}\right)$ is in the horosphere $H$. Since $a$ fixes $z$, it follows that $a n(l)$ is a geodesic with one end point at $z$ and $a n(H)$ is a horosphere based at $z$. Since $\operatorname{kan}(H)=H, k$ fixes $z$ also. This implies that $k(H)=H$. So $a n(H)=a(H)=H$.

Since $\gamma$ is parabolic and it fixes the horospheres based at $z$, it is easy to see that $\inf _{x \in X}\left(d_{\gamma}(x)\right)$ should be equal to $\lim _{x^{i} \rightarrow z}\left(d_{\gamma}(x)\right)$ for any $\left\{x^{i}\right\}$ converging to $z$. Specifically, choose $x^{i} \in l$. Note that $k$ fixes $l$ pointwise and $\inf _{x \in X}\left(d_{a}(x)\right)=d_{a}\left(x^{i}\right)$ for all $x^{i}$. Then $d\left(\operatorname{kan}\left(x^{i}\right), x^{i}\right)=d\left(\operatorname{an}\left(x^{i}\right), x^{i}\right)$ tends to $d\left(a\left(x^{i}\right), x^{i}\right)$ since $d\left(n\left(x^{i}\right), x^{i}\right)$ tends to zero. So $\inf _{x \in X}\left(d_{a}(x)\right)=\inf _{x \in X}\left(d_{\gamma}(x)\right)$. Then if $\gamma$ is a pure parabolic element, $\inf _{x \in X}\left(d_{a}(x)\right)=\inf _{x \in X}\left(d_{\gamma}(x)\right)=0$, so $a$ is the identity.

(3) Suppose it does. Let $l$ be a non-singular geodesic with end points in two opposite Weyl chambers such that $l \subset F$ for some flat $F$ whose $\partial F$ contains two opposite Weyl chambers. Then $\gamma(F) \cap F=\emptyset$, and $\gamma(l)$ is parallel to $l$ since $\gamma$ fixes those Weyl chambers. Then $l$ and $\gamma(l)$ will bound a flat which intersects $F$ along $l$. This will make $l$ singular, which is a contradiction.

In case (2) we cannot hope for better. In $H_{\mathbb{R}}^{2} \times H_{\mathbb{R}}^{2}$, let $g$ be a hyperbolic element which is in $K A$ in $I s o\left(H_{\mathbb{R}}^{2}\right)$, and let $h$ be a parabolic element in $N$ in $I s o\left(H_{\mathbb{R}}^{2}\right)$. Then $(g, 1) \cdot(1, h)$ is a mixed parabolic isometry which is in $K A N$ for $I s o\left(H_{\mathbb{R}}^{2} \times H_{\mathbb{R}}^{2}\right)$. Also in this example, $\gamma=(g, h)$ will fix two Weyl chambers, namely the ones formed by two fixed points of $g$ and a fixed point of $h$.

6.2. Invariant metric on the Furstenberg boundary. In the higher rank case, it is not easy to find an invariant metric on the Furstenberg boundary. Keep in mind that a point in the Furstenberg boundary is a Weyl chamber in the usual ideal boundary. Let $K A N$ be the Iwasawa decomposition and $X$ be the Furstenberg boundary of the symmetric space. We want to find invariant metrics on the special subsets of $X$. Let $\mathbb{Y}$ be $X$ minus the union of the ideal boundaries of flats which are fixed by some element in $A$. We claim that there is a metric on $\mathbb{Y}$ invariant under $M^{\prime} A$, where $M^{\prime}$ preserves $\mathbb{Y}$. For $x \in X-A x_{0}(\infty)$, suppose $\operatorname{Stab}_{A}(x)=\{\alpha \in$ $A \mid \alpha(x)=x\}$ is infinite. Then it is easy to see that $\operatorname{Stab}_{A}(x)$ consists of singular hyperbolic isometries as follows. Take the geodesic $l$ passing through $A x_{0}$ at $p$ with $l(\infty)=x$. Let $a \in \operatorname{Stab}_{A}(x)$. Then $a(l)$ is parallel to $l$, since $a(x)=x$ and the angle between $l$ and $A x_{0}$ is the same as the angle between $a(l)$ and $A x_{0}$. This 
shows that $a$ is a singular hyperbolic isometry. But $a$ only fixes the Weyl chambers in the flats which are left invariant by $a$. This shows that a Weyl chamber in $\mathbb{Y}$ is fixed by elements only in $M^{\prime}$. So there is a complete Riemannian metric on $\mathbb{Y}$ left invariant by $M^{\prime} A$. We will call any translate of this set (choosing a different Iwasawa decomposition) $\mathbb{Y}$. In fact, we can take $M^{\prime}$ as the normalizer of $A$ in $K$, because an element in $M^{\prime}$ leaves the flat $A x_{0}$ invariant and preserves the Weyl chamber structure of $A x_{0}$, so it leaves invariant the set of flats through $x_{0}$ stabilized by some singular elements in $A$. Then it leaves $\mathbb{Y}$ invariant.

For example, in $H_{\mathbb{R}}^{2} \times H_{\mathbb{R}}^{2}$, $\mathbb{Y}$ is equal to the torus minus two meridional circles and two longitudinal circles. Each component of $\mathbb{Y}$ is homeomorphic to $\mathbb{R}^{2}$.

Next we want to find a subset of $X$ which has an invariant metric under $N$. Let $A^{+} x_{0}(\infty)_{\text {op }}$ be the Weyl chamber opposite to $A^{+} x_{0}(\infty)$. Then by the Bruhat decomposition $N\left(A^{+} x_{0}(\infty)_{o p}\right)$ is the grand cell whose closure is the ideal boundary of the symmetric space. So there is a metric invariant under $N$ on (grand cell $/ P$ ), where $P$ is a minimal parabolic subgroup (see section 3). We call (grand cell $/ P$ ) a grand cell again, and any translate of it a grand cell (which is equivalent to choosing a different Iwasawa decomposition). Note that $M \subset K$, which fixes $A^{+} x_{0}(\infty)$ pointwise, preserves the grand cell, for the following reason. For any $n \in N, n A x_{0}$ is a flat asymptotic to $A x_{0}$ at $A^{+} x_{0}(\infty)$, so $n\left(A^{+} x_{0}(\infty)_{o p}\right)$ is the Weyl chamber in $n A x_{0}$ opposite to $A^{+} x_{0}(\infty)$. If $m \in M$, then $m n A x_{0}$ is a flat asymptotic to $A x_{0}$ at $A^{+} x_{0}(\infty)$ since $M$ fixes $A^{+} x_{0}(\infty)$ pointwise. By noting the fact that any flat asymptotic to $A x_{0}$ at $A^{+} x_{0}(\infty)$ is an image of $A x_{0}$ under $N$, we conclude that $M$ leaves the grand cell invariant. So there is a complete Riemannian metric under $M N$ on the grand cell.

For example, in $H_{\mathbb{R}}^{2} \times H_{\mathbb{R}}^{2}$, a grand cell is equal to a torus minus a meridian and a longitude, which is $\mathbb{R}^{2}$. One thing that should be noticed is that, unlike the rank one case, $M^{\prime} A(M N)$ does not fix the boundary of $\mathbb{Y}$ (a grand cell, respectively) pointwise.

In the sequel, we will assume that, unless $M$ is $X$, the developed image of a manifold $M$ modeled on $X$ lies either in $\mathbb{Y}$ or in some grand cell.

6.3. Proof of the theorem in the higher rank case. First we prove the following assertion, which is similar to Proposition 2.

Proposition 3. Let $X$ be the Furstenberg boundary of symmetric space of at least rank 2 without a Euclidean de Rham factor. Let $G$ be the isometry group of the symmetric space, which is a semisimple Lie group. Let $M$ be a closed manifold modeled on $X$, and $\rho$ the holonomy representation of $\pi_{1}(M)$ into $G$. Let $\mathcal{C}_{G}\left(\rho\left(\pi_{1}(M)\right)\right)$ be the centralizer of $\rho\left(\pi_{1}(M)\right)$ in $G$. Suppose $\phi_{t}$ is a closed non-compact one-parameter group in $\rho\left(\widetilde{\operatorname{Aut}^{0}(M)}\right) \subset \mathcal{C}_{G}\left(\rho\left(\pi_{1}(M)\right)\right)$. Suppose, unless $M$ is $X, \rho\left(\pi_{1}(M)\right)$ contains no hyperbolic isometry with a singular invariant geodesic, and $\rho(\widetilde{\operatorname{Aut}(M)})$ contains no mixed parabolic ones, and if $\rho(\widehat{\operatorname{Aut}(M)})$ contains a hyperbolic isometry, it contains a nonsingular hyperbolic isometry. Assume further, unless $M$ is $X$, that $\rho\left(\pi_{1}(M)\right)$ is not wholly contained in $K$ and the developed image lies either in $\mathbb{Y}$ or in some grand cell. Then one of the following is true.

(1) $M$ is $(G, X)$-equivalent to $X$.

(2) $\phi_{t}$ is parabolic. Then $\rho\left(\pi_{1}(M)\right)$ contains no hyperbolic isometry and $\phi_{t}$ fixes some Weyl chamber $W$. Furthermore, Aut $^{0}(M)$ is compact.

(3) $\phi_{t}$ is hyperbolic. Furthermore, Aut $t^{0}(M)$ is compact. 
Proof. Let $\left.\Gamma=\rho\left(\pi_{1}(M)\right)\right)$ and suppose $M$ is not equivalent to $X$.

Case I. $\phi_{t}$ is hyperbolic.

By the assumption, we may assume $\phi_{t}$ is non-singular. If the invariant axis $l$ of $\phi_{t}$ is non-singular, its two end points belong to two opposite Weyl chambers, $W_{0}, W_{\infty}$. Let $A x_{0}$ be the flat containing these two Weyl chambers and $G=K A N$ the corresponding Iwasawa decomposition, where $N$ fixes a unique Wely chamber $W_{\infty}$ to which one end point of the invariant geodesic $l$ belongs. If $l^{\prime}$ on $A x_{0}$ is parallel to $l$, then $\phi_{t}\left(l^{\prime}\right)$ and $\phi_{t}(l)=l$ are parallel. So if $\phi_{t}\left(l^{\prime}\right)$ is not on $A x_{0}$, the flat between $l$ and $\phi_{t}\left(l^{\prime}\right)$ will meet the flat $A x_{0}$ at $l$, which makes $l$ singular. This argument shows that $\phi_{t}\left(A x_{0}\right)=A x_{0}$ and $\phi_{t}$ leaves no other flat invariant. Since

$$
\phi_{t} \circ \gamma\left(A x_{0}\right)=\gamma \circ \phi_{t}\left(A x_{0}\right)=\gamma\left(A x_{0}\right)
$$

for $\gamma \in \Gamma$, it follows that $\gamma\left(A x_{0}\right)=A x_{0}$. So $\Gamma \subset M^{\prime} A$, where $M^{\prime}$ is the normalizer of $A$ in $K$.

Suppose $\alpha \in \rho(\widetilde{\operatorname{Aut}(M})) \subset N_{G}(\Gamma)$. Choose $\gamma^{\prime} \in \Gamma$ hyperbolic, which is guaranteed by the hypothesis that $\Gamma$ is not wholly contained in $K$, so that $\alpha \circ \gamma\left(A x_{0}\right)=$ $\gamma^{\prime} \circ \alpha\left(A x_{0}\right)$, which implies that $\alpha\left(A x_{0}\right)=A x_{0}$. Suppose $\alpha=k a n$, with $n$ not the identity. Then $n\left(A x_{0}\right) \cap A x_{0}=\emptyset$, and so $\operatorname{an}\left(A x_{0}\right) \cap A x_{0}=\emptyset$. But $k\left(x_{0}\right)=x_{0}$, so $\operatorname{kan}\left(A x_{0}\right) \neq A x_{0}$. This shows that $n$ is the identity. Then $\rho(\widetilde{A u t(M)}) \subset M^{\prime} A$.

Take a complete Riemannian metric on $\mathbb{Y}$ invariant under $M^{\prime} A$. By the assumption of the theorem, the developed image of $\tilde{M}$ is contained in $\mathbb{Y}$. Since $M$ is complete, the developing map should be a Riemannian covering onto $\mathbb{Y}$. Then $\operatorname{Aut}(M)=I s o(M)$, which is compact since $M$ is compact.

Case II. $\phi_{t}$ is parabolic.

In this case, let $A^{+} x_{0}(\infty)=W$ be the Weyl chamber which $\phi_{t}$ leaves invariant. Suppose $\gamma \in \Gamma$ is hyperbolic. Let $l$ be its invariant geodesic. Then $\phi_{t} \circ \gamma(l)=$ $\gamma \circ \phi_{t}(l)$ implies that $l$ and $\phi_{t}(l)$ are parallel, so $\phi_{t}$ leaves invariant two opposite Wely chambers to which the end points of $l$ belong. This is not possible unless $l$ is singular. Since $\Gamma$ contains no singular hyperbolic isometry, nor mixed parabolics by the hypothesis, $\Gamma \subset M N$.

Now we want to prove that $A u t^{0}(M)$ is compact.

Suppose it is non-compact. We want to show that there is no hyperbolic isometry in $\rho(\widehat{\operatorname{Aut}(M)})$, which will imply that $\rho(\widehat{\operatorname{Aut}(M)}) \subset M N$, since, by the hypothesis, there is no mixed parabolic. By the hypothesis, the developed image lies in the grand cell, which has a complete metric invariant under $M N$. This will imply that $\operatorname{Aut}(M) \subset I s o(M)$, which is compact. If $A u t^{0}(M)$ is non-compact and there is a hyperbolic isometry in it, we can form a one-parameter group $\xi_{t}$, which is hyperbolic since $\rho\left(\widetilde{A u t^{0}(M)}\right)$ is a Lie group. Then for $\gamma \in \Gamma$, since $\gamma \circ \xi_{t}(l)=\xi_{t} \circ \gamma(l)$, where $l$ is a non-singular invariant geodesic of $\xi_{t}, \gamma(l)$ and $l$ are parallel. So $\gamma$ cannot be parabolic, since it fixes two opposite Weyl chambers, which will be a contradiction to the fact that $\Gamma \subset M N$. But it can be seen directly as follows. By the same reasoning, we get then $\gamma^{n}(l)$ and $l$ are parallel for all integer $n$. If $\gamma^{n}(l)=P^{\prime} \times l$ has non-compact $P^{\prime}$, then there is a flat invariant under $\gamma$, on which it acts as a translation, so it becomes a hyperbolic isometry, which is not possible by the first part of the proof. If $P^{\prime}$ is compact $\gamma$ could be elliptic. But by the hypothesis that $\Gamma$ is not wholly contained in $K$, there should be a hyperbolic isometry, which is not permitted. 
Now we can state a similar theorem as in the rank one case.

Theorem 3. Let $M$ be a closed manifold modeled on the Furstenberg boundary $X$ of a symmetric space of rank at least two which has no Euclidean de Rham factor. Suppose, unless $M$ is $X$, the holonomy image of $\pi_{1}(M)$ contains no hyperbolic isometries with singular invariant geodesics and the holonomy image of $\widehat{A u t(M)}$ contains no mixed parabolic ones, and if it contains any hyperbolic one, it contains a non-singular hyperbolic one. Suppose further that, unless $M$ is $X$, the developed image of $M$ lies either in $\mathbb{Y}$ or in some grand cell. Then every non-singular geometric flow $\xi_{t}$ in Aut $(M)^{0}$ induces a k-torus action on $M$. In particular, if $M$ is a rational homology sphere, then there is a closed orbit in $M$.

Proof. Let $\phi_{t}=\rho\left(\tilde{\xi}_{t}\right)$ be its holonomy image in $\mathcal{C}_{A u t(X)}\left(\rho\left(\pi_{1}(M)\right)\right)$. If the flow is non-compact; then its holonomy image $\phi_{t}$ is also noncompact. Suppose $\phi_{t}$ is compact; then it is elliptic.

Case I. Let $x_{0}$ be its unique fixed point in the symmetric space. Then $\rho\left(\pi_{1}(M)\right)$ will fix $x_{0}$ also, which implies that $\rho\left(\pi_{1}(M)\right) \subset K$. Give a spherical metric on $X$ invariant under $K$. Then with respect to the pullback metric, $\pi_{1}(M)$ acts as isometries on $\tilde{M}$ to give a complete metric on $M$. Then the metric on $\tilde{M}$ is complete and $\tilde{M}$ is isometric to $X$. So $\xi_{t} \subset I s o(M)$ is compact, which is a contradiction.

Case II. Let $Z$ be the boundary of a fixed point set $Y$ of $\phi_{t}$ in $X$. If $x \neq y$ are in $Y$, then the geodesic connecting $x$ and $y$ should be left fixed pointwise by $\phi_{t}$, and so the geodesic is in $Y$, which implies that the subspace is totally geodesic, and this implies that $Z$ is the boundary of some totally geodesic subspace $S$ in the symmetric space. Then $\rho\left(\pi_{1}(M)\right)$ will leave invariant this totally geodesic subspace $S$. Then $S$ is a symmetric space again (see [9], p. 224), and the stabilizer of $S$ (which is an analytic subgroup of the isometry group of the symmetric space, [9], Theorem 7.2) in the isometry group of the symmetric space acts transitively on $S$. Then as in the proof of Theorem $2^{\prime}$, there is a metric on $X-Z$ invariant under Aut $(X-Y)$.

Note that $\phi_{t} \subset M \subset K$, where $M$ leaves $Z$ invariant. If $\operatorname{dev}(x) \in Z$ for some $x \in \tilde{M}$, since $\operatorname{dev} \circ \tilde{\xi}_{t}(x)=\phi_{t} \circ \operatorname{dev}(x)=\operatorname{dev}(x)$ and $\operatorname{dev}$ is a local homeomorphism, then $\tilde{\xi}_{t}(x)=x$. Then $\xi_{t}$ must be singular. This shows that $\operatorname{dev}(\tilde{M}) \cap Z=\emptyset$. Then, as a pair,

$$
(\rho, \operatorname{dev}):(\operatorname{Aut}(\tilde{M}), \tilde{M}) \rightarrow(\rho(\operatorname{Aut}(\tilde{M})), X-Z),
$$

we get $\operatorname{Aut}(M)=I s o(M)$, which is compact since $M$ is compact. This contradicts the fact that $\xi_{t}$ is noncompact. Hence $\phi_{t}$ must be noncompact.

Note also that if $\xi_{t}$ is noncompact, then $\rho\left(\pi_{1}(M)\right)$ is not totally contained in $K$. The proof is exactly the same as above. More precisely, if $\rho\left(\pi_{1}(M)\right)=\Gamma \subset K$, let $Z$ be the boundary of a totally geodesic subspace of the symmetric space left pointwise by $\Gamma$. If $Z=\emptyset$, given a spherical metric on $X$, by pulling back the metric to $\tilde{M}$, we get the complete metric on $\tilde{M}$ since $M$ is closed. Then $\tilde{M}=X$. Since $\Gamma$ acts freely on $X$, every element $\gamma \in \Gamma$ fixes a unique point $x_{0}$ in the symmetric space. So if $g \in N_{G}(\Gamma)$, then $g \circ \gamma\left(x_{0}\right)=\gamma^{\prime} \circ g\left(x_{0}\right)$, which implies that $g\left(x_{0}\right)=x_{0}$. So $N_{G}(\Gamma) \subset K$, and Aut $(\mathrm{M})$ is compact. This is a contradiction to the fact that $\xi_{t}$ is noncompact.

Suppose $Z$ is not empty. By the proof of Case II above, this is not possible. 


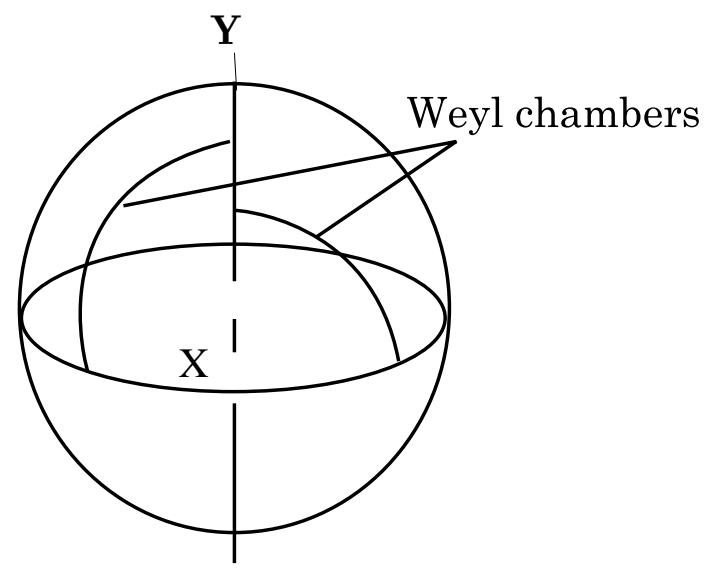

Figure 1 . Tits building of $\partial\left(H_{\mathbb{R}}^{2} \times H_{\mathbb{R}}^{2}\right)$ and the singular set

Now we can apply Proposition 3 to prove the theorem. The rest of the argument is the same as in Theorem 2.

\section{Some EXAMPLES}

In this section we give some examples to describe manifolds modeled on the Furstenberg boundary of higher rank symmetric space.

1. $H_{\mathbb{R}}^{2} \times H_{\mathbb{R}}^{2}$

(a) Holonomy is contained in the maximal compact group.

Let us consider the easiest example, which is $H_{\mathbb{R}}^{2} \times H_{\mathbb{R}}^{2}$. This space has rank 2 , and in each flat there are four Weyl chambers. Let $X=\partial H_{\mathbb{R}}^{2}, Y=$ $\partial H_{\mathbb{R}}^{2}$. Then $\partial\left(H_{\mathbb{R}}^{2} \times H_{\mathbb{R}}^{2}\right)=X * Y$. Since $I$ so $\left(H_{\mathbb{R}}^{2}\right)$ acts smoothly on each $X$ and $Y$, we can endow the smooth structure on $X * Y$ with respect to which $I$ so $\left(H_{\mathbb{R}}^{2} \times H_{\mathbb{R}}^{2}\right)$ acts smoothly. We can visualize the Tits building structure of $S^{3}$ as follows. Let $X$ be the unit circle on the $x-y$ plane in $\mathbb{R}^{3}$, and let $Y$ be the $z$-axis. Then each Weyl chamber can be identified with a quarter-circle from a point in $X$ to a point in $Y$. If we denote the quarter-circle by $x *_{t} y$ with $x *_{0} y=x, x *_{1} y=y$, the action of the isometry $(\alpha, \beta)$ can be described as

$$
(\alpha, \beta)\left(x *_{t} y\right)=\alpha(x) *_{t} \beta(x) .
$$

Let $Z_{p}$ be the group generated by the rotation of angle $\frac{2 \pi q}{p}$ acting on $H_{\mathbb{R}}^{2}$ fixing the origin in the Poincare disk. Let $Z_{2}$ be the group generated by the rotation of angle $\pi$ acting on the second factor $H_{\mathbb{R}}^{2}$. Then the group generated by $(1,1)$ acting on $X * Y$ gives the quotient manifold homeomorphic to the lens space $L(p, q)$. Then this space is certainly a rational homology 3 sphere.

In the Furstenberg boundary, what happens is the following: We can identify the Furstenberg boundary as the torus $T$ around $X$. Cut $T$ along two parallel longitudinal circles and throw away one piece (one piece is identified to the other by $Z_{2}$ action). Identify two boundary circles with twist $\frac{2 \pi q}{p}$. Then we again get a torus back. 
Note that $S O(2) \times S O(2)$ gives a toral action on $S^{3}=\partial\left(H_{\mathbb{R}}^{2} \times H_{\mathbb{R}}^{2}\right)$. The circle action $\left(e^{2 \pi i t}, e^{2 \pi i t}\right)$ is non-singular, where $e^{2 \pi i t}$ is a rotation by the angle $2 \pi t$. Indeed, this action gives the Hopf fibration of $S^{3}$.

(b) Holonomy is contained in $M A$.

We require that the developed image is contained in $\mathbb{Y}$, which is the union of four pieces homeomorphic to $\mathbb{R}^{2}$. We fix the Iwasawa decomposition in each factor, say $I s o\left(H_{\mathbb{R}}^{2}\right)=K_{1} A_{1} N_{1}, I s o\left(H_{\mathbb{R}}^{2}\right)=K_{2} A_{2} N_{2}$. Let $L_{1}$ be an open arc in $\partial H_{\mathbb{R}}^{2}$ whose end points are the two fixed points of $A_{1}$. $L_{2}$ is similarly defined. Then a component of $Y$ can be identified with $L_{1} \times L_{2}$, on which $A_{1} \times A_{2}$ acts as obvious translations. Let $(g, h),(k, l)$ be two nonsingular hyperbolic isometries. Then the group generated by them acts on $L_{1} \times L_{2}$ as $\mathbb{Z} \oplus \mathbb{Z}$, and the quotient space is the torus.

(c) Holonomy is contained in $M N$.

Let $I s o\left(H_{\mathbb{R}}^{2}\right)=K_{1} A_{1} N_{1}$ and $I s o\left(H_{\mathbb{R}}^{2}\right)=K_{2} A_{2} N_{2}$ be fixed as above. Let $x$ be the point fixed by $N_{1}$ and $y$ the point fixed by $N_{2}$. Then the grand cell is $S^{3}-\{x * Y \cup y * X\}$. In the Furstenberg boundary the grand cell is $\{X-x\} \times\{Y-y\}$, which is $\mathbb{R}^{2}$. $N_{1}$ and $N_{2}$ act as translations on each factor. As above, we can choose two parabolic isometries so that the quotient manifold is the torus.

2. $P(n, \mathbb{R})=S L(n, \mathbb{R}) / S O(n, \mathbb{R})$.

$P(n, \mathbb{R})$ is the set $\left\{X \in G L(n, \mathbb{R}) \mid X^{t}=X, \mathrm{X}\right.$ is positive definite, $\operatorname{det} X$ $=1\}$. The action of $S L(n, \mathbb{R})$ on $P(n, \mathbb{R})$ is $(A, X) \rightarrow A X A^{t}$. A Cartan subalgebra $\mathfrak{h}$ is the set of traceless diagonal matrices and the roots are $e_{i j}=$ $e_{i}-e_{j},(i \neq j)$, where $e_{i}(H)=H_{i}$ for a diagonal matrix $H=\left(H_{1}, \cdots, H_{n}\right)$. One may fix a Weyl chamber $\mathfrak{a}^{+}=\left\{\left(a_{1}, \cdots, a_{n}\right) \mid a_{1}>a_{2}>\cdots>a_{n}\right\} \subset \mathfrak{h}$ so that the corresponding fundamental system is $\left\{e_{12}, e_{23}, \cdots, e_{n-1, n}\right\}$. Note that since the walls are hyperplanes which are zero sets of $e_{i j}$, there are $n(n-1)$ Weyl chambers on each maximal flat. Furthermore a wall corresponds exactly to the case when one pair of diagonal elements are equal. Indeed it is not hard to see that the set of Weyl chambers, the Furstenberg boundary $\mathfrak{F}$, in $P(n, \mathbb{R})$ correspond to the set of flags

$$
0 \varsubsetneqq F_{1} \varsubsetneqq \cdots \varsubsetneqq F_{n-1} \varsubsetneqq F_{n}=\mathbb{R}^{n},
$$

where $F_{i}$ is a linear subspace of dimension $i$. Note here that a flat $A I$ is equal to the set of diagonal matrices with determinant 1 , and since $M$ fixes the flat $A I$ pointwise, $M=I$. Since the Furstenberg boundary $\mathfrak{F}$ is equal to $K A N / M A N=K / M$, it follows that $\mathfrak{F}=S O(n, \mathbb{R})$.

For a fixed Weyl chamber $0 \varsubsetneqq F_{1} \varsubsetneqq \cdots \varsubsetneqq F_{n-1} \varsubsetneqq F_{n}=\mathbb{R}^{n}$, the codimension one walls correspond to $F_{i}=F_{i+1}$. Also for a fixed wall $0 \varsubsetneqq F_{1} \varsubsetneqq$ $\cdots \varsubsetneqq F_{i}=F_{i+1} \varsubsetneqq \cdots \varsubsetneqq F_{n-1} \varsubsetneqq F_{n}=\mathbb{R}^{n}$, there is $\mathbb{R} P^{1}$ of Weyl chambers with this wall. Let's fix $A$ in the Iwasawa decomposition, say the exponential of $\mathfrak{h}$. Then the set of Weyl chambers left fixed by some element in $A$ consists of those Weyl chambers which share the walls of $A x_{0}$, where $x_{0}$ is the identity matrix. There are $n(n-1)$ walls in $A x_{0}$, so there are $n(n-1)$ circles $C_{1}, \cdots, C_{n(n-1)}$ such that $C_{i}$ and $C_{i+1}$ share a point (corresponding to a Weyl chamber in $\left.A x_{0}\right)$. So the set $\mathbb{Y}$ is equal to $\mathfrak{F}$ minus these circles. As we can easily see from this example that the space $\mathbb{Y}$ is not simply connected in general, $A$ does not act transitively on it, but $A$ acts freely and properly discontinuously, so there is a metric invariant under $A$ on $\mathbb{Y}$. 
The nilpotent group corresponding to the given fundamental system is the set of unipotent supertrianglular matrices. Then the orbit $N\left(A^{+} x_{0}\right)_{o p}$ is equal to $\mathfrak{F}$ minus the set of Weyl chambers which share the walls in $A x_{0}$ not equal to the walls of $\left(A^{+} x_{0}\right)_{o p}$. But this set of Weyl chambers is equal to $n(n-1)-(n-1)=(n-1)^{2}$ circles. So the grand cell is equal to $\mathfrak{F}$ minus $(n-1)^{2}$ circles. This set has a metric invariant under $N$.

\section{ACKNOWLEDGEMENT}

I want to thank Andrew Casson for his support and inspiration during my study at Berkeley. I also want to thank the referee for improvement of the theorems and better exposition of them.

\section{REFERENCES}

[1] R. Azencott and E. Wilson, Homogeneous manifolds with negative curvature I, Trans. AMS, vol 215, 1976, 323-362. MR 52:15308

[2] G. Bredon, Introduction to compact transformation groups, Academic Press, New York, 1972. MR 54:1265

[3] W. Ballmann, M. Gromov and V. Schroeder, Manifolds of nonpositive curvature, Progr. Mathematics, vol. 61, Birkhauser, 1985. MR 87h:53050]

[4] M. Druetta, Homogeneous Riemannian manifolds and the visibility axiom, Geom. Dedicata 17, 239-251 (1985).

[5] P. Eberlein and B. O'Neill, Visibility manifolds, Pac. J. Math. 46 (1973), 45-110. MR 49:1421

[6] H. Freudenthal, Oktaven, Ausnahmegruppen und Oktavengeometrie, Geom. Dedicata 19 (1985), no. 1, 7-63. MR 86k:17018b

[7] J. C. Harrison, $C^{2}$ counterexamples to the Seifert conjecture, Topology 27 (1988), 249-278. MR 89m:58167

[8] J. Heber, On the geometric rank of homogeneous spaces of nonpositive curvature, Invent. math. 112, 151-170 (1993). MR 94a:53082

[9] S. Helgason, Differential geometry, Lie groups, and symmetric spaces, Academic Press, 1978. MR 80k:53081

[10] H. Hofer, Pseudoholomorphic curves in symplectizations with applications to the Weinstein conjecture in dimension three, Invent. Math. 114, 515-563 (1993). MR 94j:58064

[11] Y. Kamishima, Geometric Flows on Compact Manifolds and Global Rigidity, Topology 35, 439-450, 1996.

[12] I. Kim, Marked length rigidity of rank one symmetric spaces and their product, to appear in Topology.

[13] K. Kuperberg, A smooth counterexample to the Seifert conjecture, Ann. of Math. (2) 140 (1994), 723-732. MR 95g:57040

[14] G. D. Mostow, Strong rigidity of locally symmetric spaces, Ann. of Math. Stud., vol. 78. Princeton Univ. Press, Princeton, NJ, 1973. MR 52:5874

[15] P. Pansu, Métriques de Carnot-Carathéodory et quasiisométries des espaces symétriques de rang un, Ann. Math. 129 (1989), 1-60. MR 90e:53058

[16] P. A. Schweitzer, Counterexample to the Seifert conjecture and opening closed leaves of foliations, Ann. of Math. (2) 100 (1974), 386-400. MR 50:8557

[17] W. P. Thurston, Three Dimensional Geometry and Topology, The Geometry Center, unpublished manuscript.

Department of Mathematics, Korea Advanced Institute of Science and Technology, 373-1 Kusong-Dong Yusong-Ku, TAejon 305-701, Korea

E-mail address: inkang@mathx.kaist.ac.kr 